\title{
The Discrepancy in Galaxy Rotation Curves
}

\author{
Roy Gomel ${ }^{1, *}$ and Tomer Zimmerman ${ }^{1}$ \\ (1) School of Physics and Astronomy, Tel Aviv University, Israel \\ Corresponding: roygomel@post.tau.ac.il
}

\begin{abstract}
In this work we try a new approach for dealing with the discrepancy between observed galaxy rotation curves and theoretical predictions. This new approach does not involve any changes in the current fundamental laws of nature or the addition of dark halos. Rather, it is based on the following single assumption: the observed velocities presented in rotation curves are not given relative to the galaxies' local inertial frames. Another way of putting it down: fictitious f orces, w hich a rise i $\mathrm{n} n$ on-inertial frames, should be taken into account when constructing a theoretical rotation curve. It turns out that this single assumption is sufficient in order to establish a robust model for fitting rotation curves. Applying the new model on a sample of more than 30 galaxies provides very promising results.
\end{abstract}

Keywords: dark matter; reference frames; galaxies

\section{INTRODUCTION}

A Galaxy Rotation Curve (RC) describes the rotational velocities of objects in a galaxy as a function of their radial distance from the galactic center. One of the pioneers in this field was the American astronomer Horace Babcock who measured Andromeda's RC in 1939 and unexpectedly found that the orbital velocities of its stars increase with distance (Babcock 1939). This result was surprising due to the fact that Andromeda's mass distribution points out that the rotational velocities should actually decrease with distance. An illustration of this discrepancy (based on more modern data) is shown in Fig. 1.

During the seventies a meaningful progress was made when Vera Rubin constructed RC's for a wide range of galaxies (Rubin, Ford \& Thonnard 1980). Looking at the measured data she found that none of the rotation curves were declining with the radial distance. Some RC's showed a monotonically increasing trend over the entire range of distances while others showed an asymptotically flat behavior. Her published work paved the way to the following conclusion: RC's cannot be explained only by the visible matter. A large amount of mass (5-10 times the mass of visible matter) should be found mainly in the outer parts of a galaxy in order to explain the measured RC's. This kind of missing mass was named "Dark Matter". Many studies have been conducted over the years in order to reveal the essence of this matter. Today's common view assumes that dark matter is composed of exotic particles that cannot interact with their surroundings through electromagnetic forces.

Some researchers didn't feel comfortable with the mysterious invisible dark matter and proposed alternative theories. A well known theory of this kind has been proposed by Mordehai Milgrom 


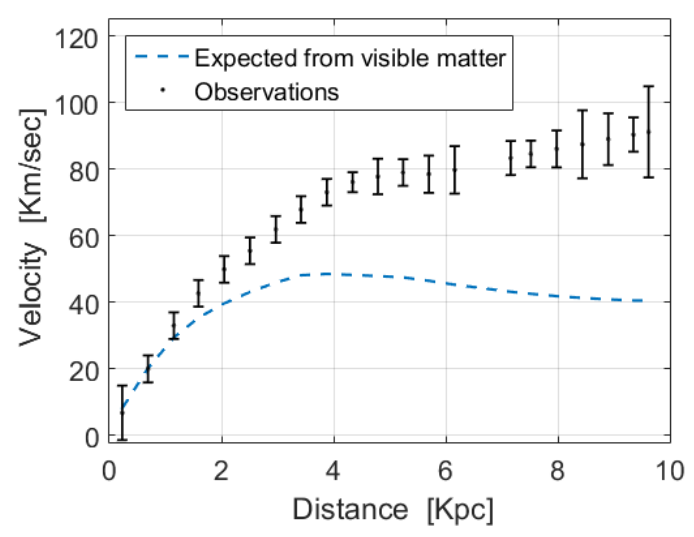

Fig. 1: A galaxy measured RC (black error bars) and a predicted RC (blue dashed line) are plotted. The data (NGC 100) was taken from McGaugh's Data Pages (McGaugh 2017).

(Milgrom 1983). His theory modifies Newton's laws of motion in such a way that there is no need for extra matter and the predicted curves are always asymptotically flat. A large amount of RC's were fitted using his theory. The Modified Newtonian Dynamics (MOND) can be surely regarded as a worthy competitor of dark halos in the field of galaxy rotation curves. However, it still faces tough theoretical challenges.

The aim of this current work is to propose an explanation for the said discrepancy without the need for extra matter or the need of changing Einstein's General Relativity and Newton's laws. In section 2 we introduce the new explanation and derive a model for dealing with the discrepancy. In sections 3, 4 and 5 we use this model in order to construct RC's: in section 3 we construct RC's in a schematic approach, in section 4 we fit RC's of several different galaxies using an analytical approach and in section 5 we fit RC's for a large amount of galaxies using a numerical approach. In section 6 we demonstrate that our approach leads to an effective halo which is similar to the current common dark halos. In section 7 we conclude and discuss some future directions.

\section{DERIVING A NEW MODEL}

It is well known in physics that fundamental quantities like "position" and "time" (and other quantities that are derived from them as well, like "velocity") are relative and have a meaning only relative to a well defined coordinate system. Newton's laws of motion, which are used successfully to explain a huge amount of physical phenomena, cannot be treated as "absolute" either. These laws are valid only relative to coordinate systems which we call "Inertial" (Einstein 1920). Therefore, if we wish to compare between an observed quantity (e.g. "velocity") and its Newtonian prediction, we have to make sure that the observed quantity is given relative to an Inertial frame of reference. If the observed quantity is not given relative to an inertial frame of reference, then fictitious forces should be added to the model.

Let us focus on the RC's problem. As noted before, an RC of a galaxy is a plot of measured rotational velocities vs. distance from galactic center. When these rotational velocities are being predicted by using Newton's laws of motion, a discrepancy is revealed. In order to resolve the discrepancy, two main approaches were taken: 
1. Newton's laws do not apply in these cases. A modification of the laws (and of general relativity) is required.

2. Newton's laws do apply in these cases but an extra amount of matter should be taken into account.

We propose a third possibility:

3. Newton's laws do apply in these cases and no extra matter should be taken into account. However, the measured velocities of each and every galaxy (e.g. the black data points in Fig. 1) are systematically not given relative to the galaxies local inertial frames. In those non-inertial frames, fictitious forces arise, and must be taken into account as well.

In what follows, we explain the essence of this assumption and use it in order to derive a corrected rotation curve.

First, let us introduce the frame $K$, which is assumed to be Inertial. That is, a frame of reference relative to which Newton's laws of motion apply for the purpose of describing the motions of bodies in a specific galaxy. Without loss of generality, one can always arrange this frame in such a way that its $x-y$ plane will coincide with the galactic plane and its origin will coincide with the galactic center of this specific galaxy. The reason for this is as follows: if Newton's laws are to be valid relative to that frame, then, assuming a central force problem, each body will preserve its plane of motion. Therefore the whole galactic plane will be static relative to that frame. It is now only a matter of definition to call this plane "the $x-y$ plane".

We now introduce another system of coordinates, $K^{\prime}$, relative to which the measured rotational velocities are given. It turns out that system $K^{\prime}$ can also be chosen such that its $x^{\prime}-y^{\prime}$ plane coincides with the galactic plane and its origin coincides with the galactic center (see Appendix A). However, system $K^{\prime}$ is constrained: the line-of-sight connecting the observer and the specific galaxy must be static relative to that frame (again, see Appendix A). With this arrangement, one has no basis to assume the coincidence of $K$ and $K^{\prime}$. There is a possible relative motion between the two systems: an angular velocity of one system around the $z$-axis of the other (see Fig. 2). In general, this angular velocity can change with time. In this work, however, we have chosen to restrict ourselves to the simplest case of constant angular velocities. Without any further information, this constant angular velocity is unknown. Therefore, at this work, it will be regarded as a free parameter used to find the assumed inertial system $K$. Before proceeding, it's important to emphasize: each galaxy is characterized by a unique $K^{\prime}$, and in general different $K^{\prime}$ frames coming from different galaxies are not necessarily fixed one with respect to the others.

In accordance with the previous definitions, our goal is basically to make a prediction for the rotational velocities $v_{K^{\prime}}$. For this purpose, though, a relation between the velocities $v_{K^{\prime}}$ and $v_{K}$ should be found. By using such a relation and the known models for $v_{K}$, the velocities $v_{K^{\prime}}$ could be extracted. Although this is quiet straightforward, it would be beneficial to make the longer derivation, starting from the relation between the accelerations. Given a frame of reference $K^{\prime}$, rotating at a constant angular velocity $\omega$ relative to an Inertial frame $K$, the relation between the accelerations in the two frames is given by:

$$
\mathbf{a}_{\mathbf{K}^{\prime}}=\mathbf{a}_{\mathbf{K}}-2 \boldsymbol{\omega} \times \mathbf{v}_{\mathbf{K}^{\prime}}-\boldsymbol{\omega} \times(\boldsymbol{\omega} \times \mathbf{r})
$$

Where $\mathbf{a}_{\mathbf{K}^{\prime}}$ is the acceleration of a body relative to $K^{\prime}, \mathbf{a}_{\mathbf{K}}$ is the acceleration of that body relative to $K, \boldsymbol{\omega}$ is the constant angular velocity at which system $K^{\prime}$ revolves relative to system $K, \mathbf{v}_{\mathbf{K}^{\prime}}$ is the velocity of the body relative to $K^{\prime}$ and $\mathbf{r}$ is the position of the body. 


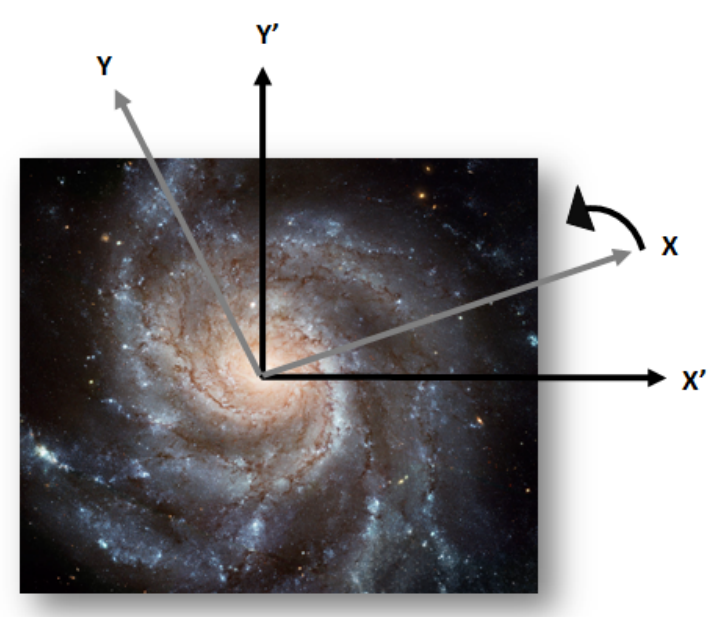

Fig. 2: The inertial system of coordinates $(K)$ revolves relative to the non-inertial one $\left(K^{\prime}\right)$. The measured velocities presented in RC's are valid only with respect to $K^{\prime}$ by definition.

The first expression, $2 \boldsymbol{\omega} \times \mathbf{v}_{\mathbf{K}^{\prime}}$, is known as the Coriolis force, and the second expression, $\boldsymbol{\omega} \times(\boldsymbol{\omega} \times \mathbf{r})$ is known as the centrifugal force. Now, in our case we would like to define a positive angular velocity in the opposite direction, as can be seen in Fig. 2. Therefore, taking $\omega \rightarrow-\omega$ and assuming that the body performs a counterclockwise uniform circular motion leads to the following relation in the $\hat{r}$ direction:

$$
-\frac{v_{K^{\prime}}^{2}}{r}=-\frac{v_{K}^{2}}{r}-2 \omega v_{K^{\prime}}+\omega^{2} r
$$

Where $v_{K^{\prime}}\left(v_{K}\right)$ is the rotational velocity of the body relative to $K^{\prime}(K), \omega$ is the constant angular velocity at which system $K$ revolves around the $z^{\prime}-$ axis of system $K^{\prime}$ and $r$ is the radius of the circular motion.

At this stage, a useful sanity check could already be done. We will recall that when adding a dark halo (in order to resolve the discrepancy), an extra force in the inward direction is produced. Therefore, in our case, we have to make sure that the two fictitious forces together could also act in the inward direction. Looking at Eq. (2), one can see that this condition will be satisfied if $\omega^{2} r<2 \omega v_{K^{\prime}}$, or $0<\omega<\frac{2 v_{K^{\prime}}}{r}$. For a given galaxy and a given observed rotation curve $v_{K^{\prime}}(r)$, this inequality sets limits on $\omega$. Within these limits, an inward force arises in $K^{\prime}$ at any radius, as required. Whether this extra inward force could actually produce good fits to the observed data is the subject of the next sections.

Now, the last stage is solving Eq. (2). This gives:

$$
v_{K^{\prime}}=v_{K}+\omega r
$$

All the cases examined and shown in the next sections are based on this simple correction to the predicted velocities.

\section{A SCHEMATIC DEMONSTRATION OF THE NEW APPROACH}

Trying to predict an RC, one should first model the galaxy's mass distribution. This is often done by dividing the galaxy into its different components (i.e. Bulge, Stellar disk, Gaseous disk and Dark halo) and calculating the contribution of each component to the rotational velocity. The velocity is then given by: 


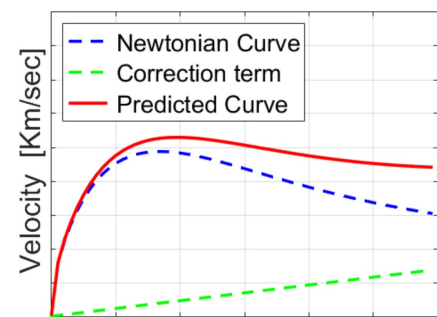

Distance $[\mathrm{Kpc}]$

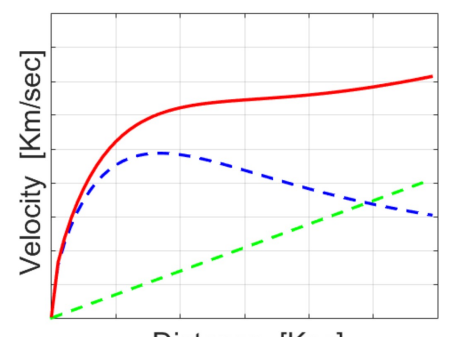

Distance $[\mathrm{Kpc}]$

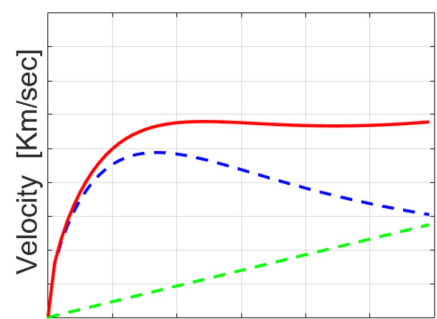

Distance $[\mathrm{Kpc}]$

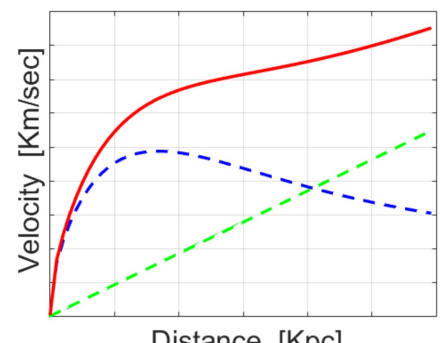

Distance $[\mathrm{Kpc}]$

Fig. 3: Four schematic RC's. The blue line represents the Newtonian rotation curve of a typical galaxy, the green line represents the linear correction term and the red line represents the predicted curve. One can see four trends: a slowly declining RC, A flat RC, A slowly rising RC and a rising RC. All four trends were actually observed in measured RC's.

$$
V(r)=\sqrt{v_{b}^{2}(r)+v_{d}^{2}(r)+v_{g}^{2}(r)+v_{d a r k}^{2}(r)}
$$

Where $V(r)$ is the predicted RC, $v_{b}(r), v_{d}(r), v_{g}(r)$ are the contributions of the visible components and $v_{\text {dark }}(r)$ is the contribution of the dark halo.

The derivation of the different velocities from their corresponding mass distributions assumes the validity of Newton's laws. Therefore, the total rotational velocity $V(r)$, is a valid prediction only relative to an Inertial frame of reference.

Our proposition, however, states that the observed values might not be given relative to an Inertial frame. Therefore, a correction term should be added to the predicted RC, $V(r)$.

The rotational velocity will be given now by:

$$
V(r)=\sqrt{v_{b}^{2}(r)+v_{d}^{2}(r)+v_{g}^{2}(r)}+\omega r=V_{\text {inertial }}(r)+\omega r
$$

Where $\omega$ is the constant angular velocity in which the inertial system revolves relative to the observational system, as was explained in the previous section. Note that a dark halo component is not required when taking this approach.

Let us now demonstrate schematically the behavior of the function $V(r)$. For this purpose, we have drawn four different RC's. We have chosen a typical $V_{\text {inertial }}(r)$ to be the same in all four cases and changed only the value of $\omega$, which is the slope of the linear correction term. The results are shown in Fig. 3. One can notice that the behavior of the schematic RC's is consistent with the behavior of actual measured RC's. 


\section{APPLYING THE NEW APPROACH USING AN ANALYTICAL MASS MODEL}

As mentioned in the previous section, when trying to predict an $\mathrm{RC}$, the galaxy's mass distribution should first be modeled. For this purpose, we used the most common analytical model. The model includes three different components (or parts of them): a spherical bulge, a stellar disk and a gaseous disk. Let us introduce shortly the model's ingredients.

- The spherical bulge represents the central group of stars found in most spiral galaxies. We used an Hernquist density profile of the form:

$$
\rho(r)=\frac{M_{b}}{2 \pi r_{b}^{3}} \frac{1}{\frac{r}{r_{b}}\left(1+\frac{r}{r_{b}}\right)^{3}}
$$

where $\rho(r)$ is the volume mass density, $r$ is the radial distance from the galactic center, and $r_{b}$ is the sphere characteristic scale length. Assuming a circular motion results in the following velocity (Hernquist 1989):

$$
v_{b}^{2}=\frac{G M_{b} r}{\left(r+r_{b}\right)^{2}}
$$

- The stellar disk represents the flat part of the galaxy which continues beyond the central bulge and is mainly composed of the galaxy's stars. It is modeled as a two dimensional disk with a surface mass density of the form:

$$
\Sigma(r)=\Sigma_{0} e^{-\frac{r}{r_{d}}}
$$

where $\Sigma(r)$ is the surface mass density, $\Sigma_{0}$ is the central mass density, $r$ is the distance from the disk center, and $r_{d}$ is a characteristic scale length. The disk mass is given by:

$$
M_{d}=2 \pi r_{d}^{2} \Sigma_{0}
$$

Assuming circular motion results in the following velocity (Freeman 1970):

$$
v_{d}^{2}=4 \pi G \Sigma_{0} r_{d} y^{2}\left[I_{0}(y) K_{0}(y)-I_{1}(y) K_{1}(y)\right]
$$

where $y \equiv \frac{r}{2 r_{d}}$ and $I, K$ are the Modified Bessel Functions of first and second kind.

- The gaseous disk, like the stellar one, represents the flat part of the galaxy around the central bulge that is mainly composed of the galaxy gas and dust. In many cases a Gaussian density distribution can be assumed, yielding:

$$
\sigma(r)=\sigma_{0} e^{-\frac{r^{2}}{2 r_{g}^{2}}}
$$

where $\sigma(r)$ is the surface mass density, $\sigma_{0}$ is the central mass density, $r$ is the distance from the disk center, and $r_{g}$ is a characteristic scale length. The disk mass is given by:

$$
M_{g}=2 \pi r_{g}^{2} \sigma_{0}
$$

Assuming circular motion results in the following velocity (Toomre 1963):

$$
v_{g}^{2}=\frac{\sqrt{\pi}}{2 \sqrt{2}} G M_{g} \frac{r^{2}}{r_{g}^{3}} F\left(1.5,2,-\frac{r^{2}}{2 r_{g}^{2}}\right)
$$

where $\mathrm{F}$ is the Generalized Hyperbolic Function. 

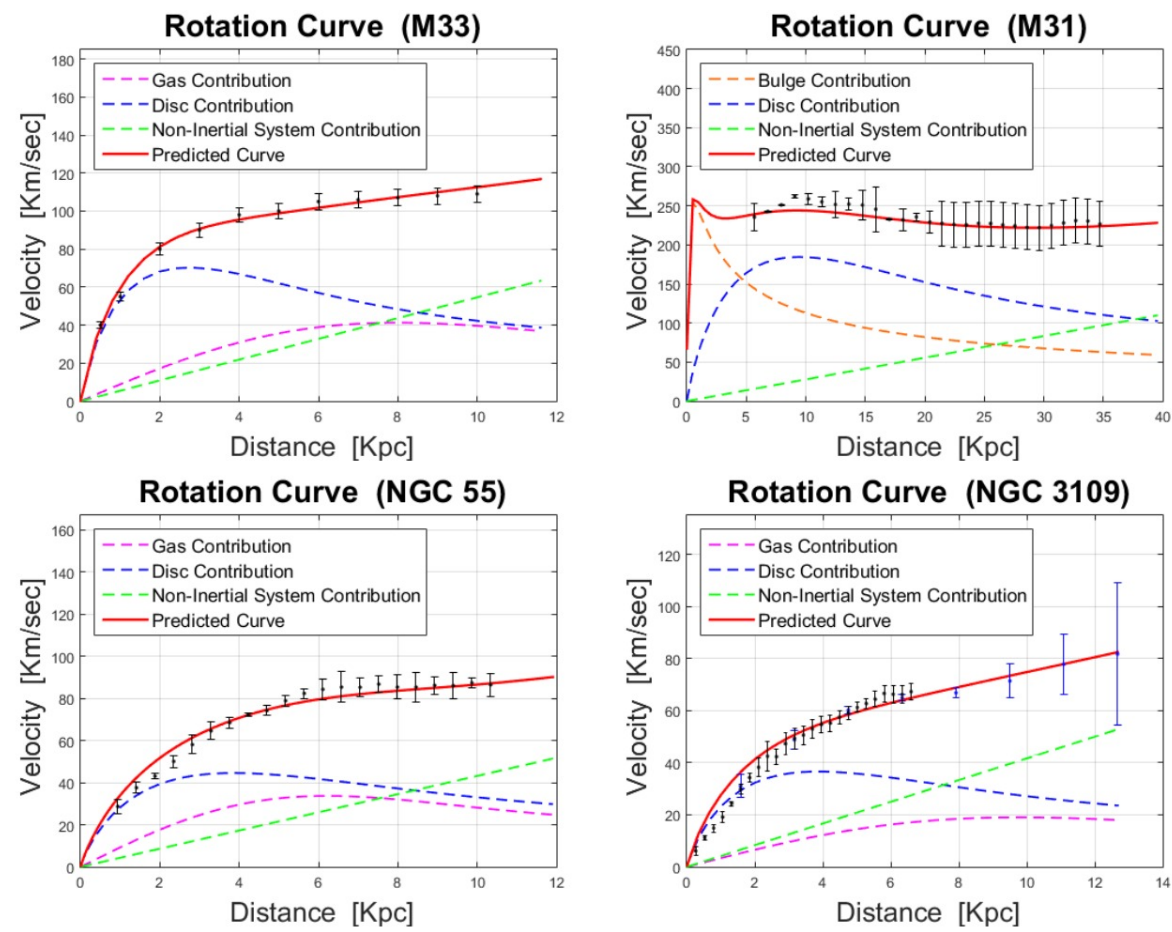

Fig. 4: RC's of four different galaxies are presented. In each figure, one can see the measured values (black error bars) together with the predicted curve (red line). The blue dashed curve represents the stellar disk contribution $v_{d}$, the magenta dashed curve represents the gas contribution $v_{g}$, the orange dashed curve represents the bulge contribution $v_{b}$ and the green dashed curve represents the $\omega r$ correction. Top left panel: Rotation Curve of M33 (Corbelli \& Salucci 2000), Top right panel: Rotation Curve of M31 (Carignan et al. 2006), Bottom left panel: Rotation Curve of NGC 55 (Puche, Carignan \& Wainscoat 1991), Bottom right panel: Rotation Curve of NGC 3109 (Jobin \& Carignan 1990; Carignan et al. 2013)

The derivation of the velocities $v_{b}, v_{d}, v_{g}$ from their corresponding mass distributions assumes the validity of Newton's laws. Therefore, these velocities are valid predictions only relative to inertial reference frames. Specifically, relative to the inertial system $\mathrm{K}$, whose $x-y$ plane coincides with the galactic plane. The total (inertial) velocity of an object lying on the galactic plane and located at a distance $r$ from the origin, is then given by:

$$
V_{\text {inertial }}(r)=V_{K}(r)=\sqrt{v_{b}^{2}(r)+v_{d}^{2}(r)+v_{g}^{2}(r)}
$$

If we wish to describe the object's motion relative to system $\mathrm{K}^{\prime}$ (the frame of reference in which measurements are given) we should add the correction term $\omega r$ to the velocity $V_{K}(r)$, as was explained in previous sections.

This method was used for analyzing four different galaxies. For each galaxy $V_{K}(r)$ was calculated according to Eq. (14), using the characteristic masses and scale lengths of the specific galaxy. Then the value of $\omega$ was set to give the best fit to the measured $\mathrm{RC}$ using the least squares method. The values of the galaxies' characteristic masses and scale lengths are summarized in Table 1. The measured RC's together with those predicted by our model are presented in Fig. 4. Below the figure we indicate the references from which the measured data was taken. 
Table 1 The model parameters (masses and scale lengths) for the different galaxies

\begin{tabular}{ccccc}
\hline Galaxy & NGC 3109 & NGC 55 & M33 & M31 \\
\hline Ref & (Carignan et al. 2013) & (Puche, Carignan \& Wainscoat 1991) & (Corbelli \& Salucci 2000) & (Geehan et al. 2006) \\
\hline$r_{b}[$ Kpc $]$ & - & - & - & 0.61 \\
$r_{d}[$ Kpc $]$ & 1.8 & 1.8 & 1.27 & 4.4 \\
$r_{g}[$ Kpc $]$ & 5.5 & 3.5 & 4.5 & - \\
$M_{b}\left[10^{9} M_{\odot}\right]$ & - & - & - & 33 \\
$M_{d}\left[10^{9} M_{\odot}\right]$ & 1.45 & 2.15 & 3.77 & 90 \\
$M_{g}\left[10^{9} M_{\odot}\right]$ & 0.7 & 1.4 & 2.7 & - \\
$\omega\left[10^{-16} \mathrm{rad} / \mathrm{sec}\right]$ & 1.35 & 1.4 & 1.77 & 0.9 \\
\hline
\end{tabular}




\section{APPLYING THE NEW APPROACH USING NUMERICAL MASS MODEL}

The next step, naturally, is trying to fit RC's for a large number of galaxies using modern techniques. In contrast to the calculations given in the previous section, the Newtonian contribution, $V_{K}(r)$, is now being calculated numerically by using the observed galaxy's light distribution. A detailed description of this process can be found in Sanders \& McGaugh Annual Review (Sanders \& McGaugh 2002). Unless a bulge is present, a single parameter is required when using this method. This parameter is the Mass to Light ratio $(\mathrm{M} / \mathrm{L})$, which is assumed to be constant for a given galaxy. The Newtonian velocity is then given by:

$$
V_{\text {inertial }}(r)=V_{K}(r)=\sqrt{(M / L) \cdot v_{d i s k}^{2}(r)+v_{g}^{2}(r)}
$$

Where $M / L$ is the free parameter, $v_{d i s k}(r)$ is the known stellar disk contribution (assuming $M / L=1)$ and $v_{g}(r)$ is the known gaseous disk contribution.

In this work, the galaxies' data (i.e. the observed RC's and the model contributions $v_{\text {disk }}(r)$ and $v_{g}(r)$ for each individual galaxy) was kindly given by Prof. Stacy McGaugh. The data can be found in McGaugh's Data Pages (McGaugh 2017). This is the same sample used by McGaugh in his Baryonic Tully-Fisher work (McGaugh 2005). For the sake of simplicity, we selected only galaxies without a bulge contribution. This results in a total amount of 34 galaxies.

As was shown in previous sections, an $\omega r$ term should be added to the Newtonian term in order to predict an observed RC. Therefore, a model with 2-parameters $(M / L$ and $\omega)$ has been used together with the Least-Squares method in order to fit each individual RC. A fair selection of our fits to RC's is given in Fig. 5. The rest of the fits and a table summarizing the best-fit parameters are given in Appendix B. Correlations between $\omega$ and other galaxy parameters are plotted in Fig. 6.

The first nice outcome is the consistency of the fits with Renzo's experimental law (Sancisi 2004). This law states that every time there is a feature in the radial light distribution, the rotation curve shows a corresponding feature. In our case, the inertial contribution, $V_{K}(r)$, was directly derived from the light (and gas) distributions. Indeed, adding only a straight line to $V_{K}(r)$ would preserve every bump and feature. This nice result can be seen in the outer parts of NGC 5033 for example.

Another natural result is the dominance of the Newtonian curve in the inner regions of the RC's. It can be seen that in the inner regions, where the linear term is still small, the Newtonian term almost fits the data. Actually, it is similar to the maximum disk approach: there, in the inner regions, the baryonic curve fits the data and the dark halo contribution is still small.

The next point deals with the $M / L$ values. Looking at Table 2 (Appendix B) one can notice that the values obtained for the $M / L$ 's are quiet reasonable. The median value of the population is 1.5 $\left[M_{\text {sun }} / L_{\text {sun }}\right]$ while the median absolute deviation is $0.5\left[M_{\text {sun }} / L_{\text {sun }}\right]$.

The last point deals with the values of our new parameter, $\omega$. These values demonstrate a very interesting situation. First, we have got only positive $\omega$ 's in our fits. It means that the local inertial frame (of each and every galaxy) is "dragged" relative to the observational frame in the same direction as the direction of the revolving matter in that galaxy. Second, as can be seen in Fig. 6, the values of $\omega$ correlates with other galaxy parameters. In the left panel one can see that the larger the radius of a galaxy (represented here by $r_{\max }$, the last point of the RC), the smaller the value of $\omega$. In the right panel one can see a similar trend (although weaker) with the galaxy's total mass. These results might serve as a hint for further investigations of the subject. 

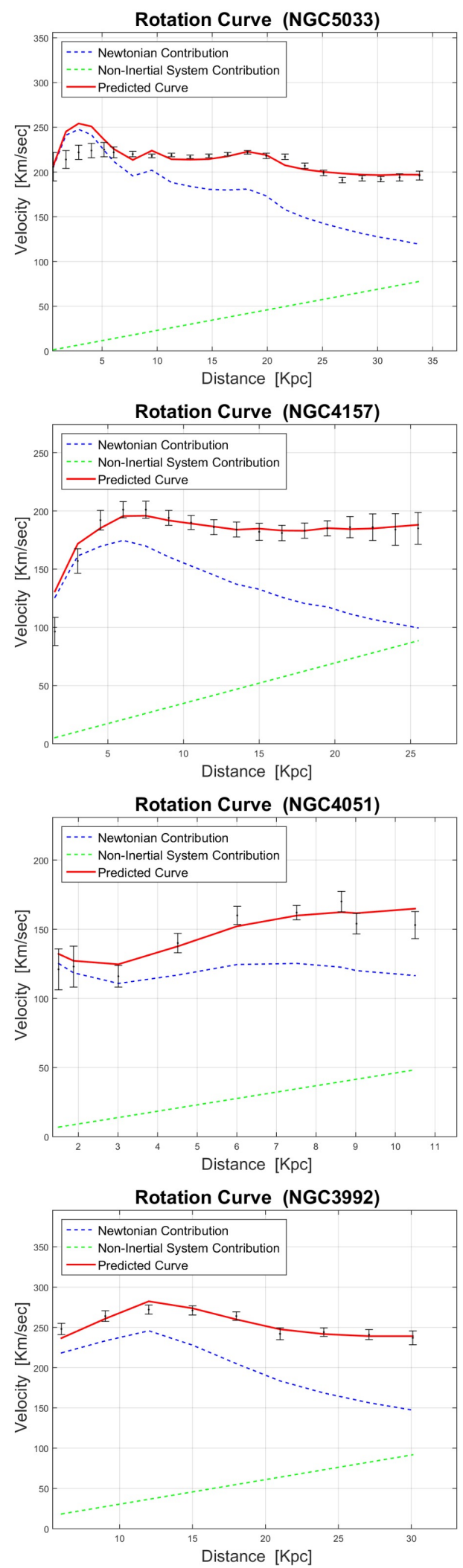
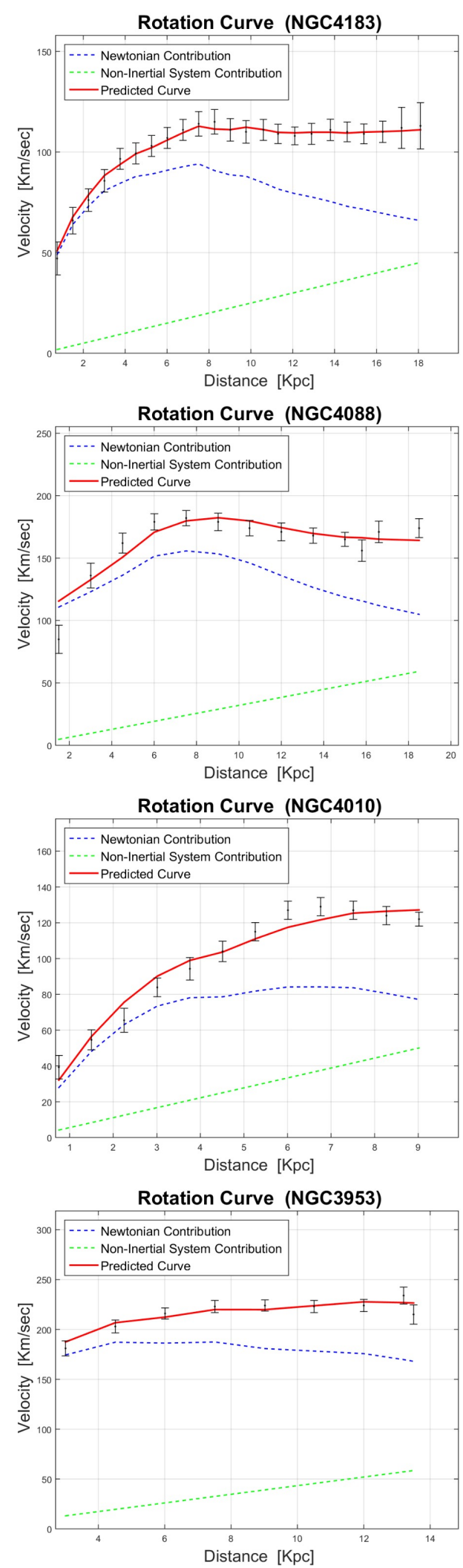

Fig. 5: RC's for the different galaxies are presented. In each panel, one can see the measured values (black error bars) together with the predicted curve (red line). The blue dashed curve corresponds to the Newtonian term $v_{k}(r)$ while the green dashed line corresponds to the correction term $\omega r$. 

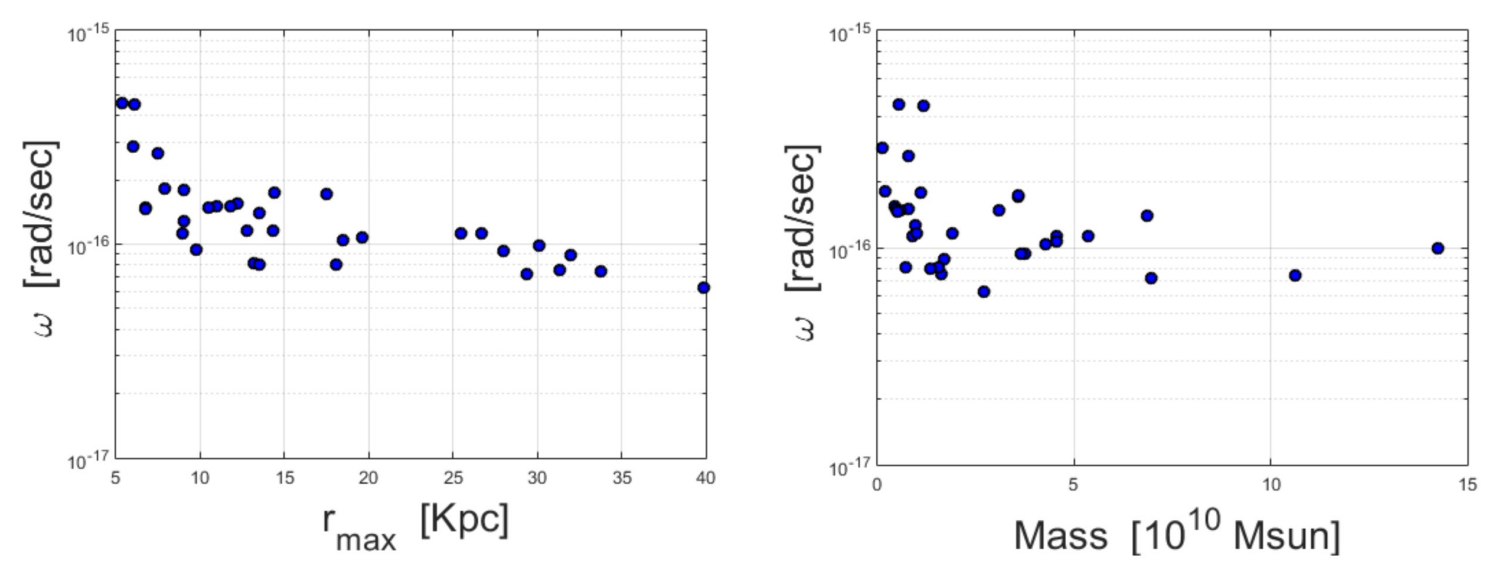

Fig. 6: Left panel: a scatter plot of $\omega$ (obtained from the best fit) vs $r_{\max }$ (the last point of the rotation curve). Right Panel: a scatter plot of $\omega$ vs the galaxy's mass. Both plots were produced using the 34 galaxies sample analyzed in this work.

\section{THE EFFECTIVE DARK HALO}

It is well known that dark halos are used successfully to fit all sorts of RC's. Therefore, it would be beneficial to check whether our approach can lead to an effective dark halo which is somehow similar to the common dark halo profiles used today.

For this purpose, equality between the two different formulas of $V_{c}(r)$ is required (see section 3 ). This condition is given by:

$$
\sqrt{V_{\text {baryonic }}^{2}(r)+V_{\text {dark }}^{2}(r)}=V_{\text {inertial }}(r)+\omega r
$$

Where $V_{\text {baryonic }}(r)$ and $V_{\text {inertial }}(r)$ refer exactly to the same thing: the circular velocity produced by the baryonic matter as seen from an inertial frame of reference.

Extracting $V_{\text {dark }}(r)$ from Eq. (16) gives:

$$
V_{\text {dark }}(r)=\sqrt{\omega^{2} r^{2}+2 \omega r V_{\text {baryonic }}(r)}
$$

Where $V_{\text {dark }}(r)$ is the circular velocity of a body orbiting around an effective dark halo, i.e. a dark halo which produces the same velocity field (and gravitational field) as fictitious forces do.

Assuming a spherically symmetrical effective halo, the density distribution can also be calculated. A short derivation gives:

$$
\rho_{\text {dark }}(r)=\frac{\omega}{4 \pi G}\left(3 \omega+4 \frac{V_{\text {baryonic }}(r)}{r}+2 V_{\text {baryonic }}^{\prime}(r)\right)
$$

As can be seen in Equations 17-18, the effective halo is characterized by some unique features. The first one is the number of free parameters. While common dark halo profiles use two or three free parameters, the effective halo uses only one, namely $\omega$ (we disregard the $M / L$ parameter hiding in $V_{\text {baryonic }}(r)$ in purpose, because it has to be set in any case, also when common halos are used). The second feature is the halo dependence on baryonic matter. While common profiles do not include such 

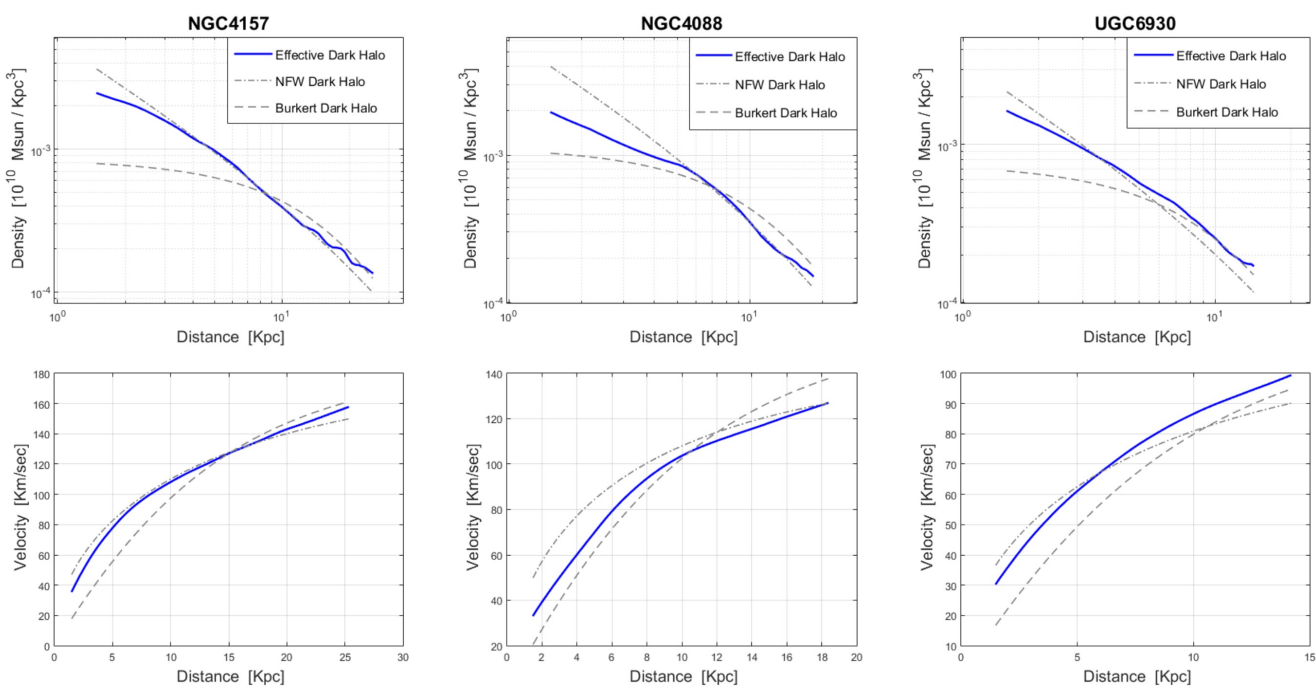

Fig. 7: Density and velocity distributions of spherical halos. The Burkert and NFW halos have two parameters: Mvir (the virial mass in $\left[10^{10}\right.$ Msun]) and c (the concentration parameter which is unitless). The effective halo has only one parameter, which is $\omega$, given in $\left[10^{-16} \mathrm{rad} / \mathrm{sec}\right]$. Left Panels: NGC 4157 (NFW: Mvir $=170, \mathrm{c}=5$; Burkert: Mvir $=120, \mathrm{c}=12$; Effective halo: $\omega=1.12$ ). Center Panels: NGC 4088 (NFW: Mvir = 70 , c = 7; Burkert: Mvir = 70, $\mathrm{c}=13.5$; Effective halo: $\omega=1.04$ ). Right Panels: UGC 6930 (NFW: Mvir = 40, c = 5; Burkert: Mvir = 30, c = 11.5; Effective halo: $\omega=1.17$ ).

a link, here it comes naturally. This link between dark (or total) mass distribution and baryonic mass distribution has been discussed already in the literature, especially by Swaters (Swaters et al. 2012). Their results support the idea that dark and baryonic distributions are intimately connected.

Obviously, the next step is plotting the effective halo together with some common halo profiles, such as NFW (Navarro, Frenk \& White 1997) or Burkert (Burkert 1995). However, the dependence of the effective halo on $V_{\text {baryonic }}(r)$ compels us to do so only per given galaxy. Moreover, while common halos continue as long as $R_{v i r}$, our halo is defined only within the baryonic region, that is, to the last point of the rotation curve.

In Fig. 7 we use three different galaxies in order to compare between a Burkert dark halo, an NFW dark halo and our effective halo. For each galaxy we draw the density distribution and the circular velocity distribution produced by each halo. Drawing the Burkert and the NFW distributions is done by setting their two free parameters. Drawing the effective halo is done by setting $\omega$ and using $V_{\text {baryonic }}(r)$. For each galaxy, the parameters that best fitted the data were chosen. In the case of the effective halo, it is the best-fit $\omega$ obtained in the previous section. In the case of the Burkert or NFW halos, those are the two free parameters that best fitted the data using a fixed M/L (the M/L found in the previous section).

Looking at each panel of Fig. 7, the main result is of course the resemblance between the distributions of the effective halo and the dark halos. In other words, we've found that fictitious forces, stem from a simple coordinates transformation, can reproduce (in general) the behavior of dark halos. Going more into details, one can notice that the effective halo density distribution in the inner region is less steeper than NFW. In the central region it pretty much follows the NFW behavior, while in the outer regions it has a "tail". A more comprehensive examination of the subject (including more galaxies) is not included in the scope of this work. 


\section{SUMMARY AND FUTURE DIRECTIONS}

Two main approaches were taken so far in order to deal with the discrepancy in galaxy rotation curves. Either change the underlying laws of physics or add more mass to the detectable mass distribution. In both cases the different dynamics result in different rotational velocities. In the scope of this work, however, we assumed that Newton's laws and the detectable mass distribution are sufficient for predicting the orbital motions in a galaxy. We rather proposed that the observed velocities are not given relative to the galaxy's local inertial frame, thereby generating the discrepancy. We have demonstrated that a single degree of freedom is necessary (and sufficient) in order to represent the relative motion between the observational frame and the local inertial frame. Relying on this degree of freedom, we developed a model which predicts the observed (non-inertial) rotational velocities. Applying the model analytically and numerically ended up with promising results.

An interesting outcome of this work was discussed in the end of section 5: only positive angular velocities were obtained from the fitted rotation curves. It means that the inertial frame of each galaxy is "dragged" relative to the observational frame in the same direction as the direction of the revolving matter. Therefore, the rotating matter in a galaxy seems to play a role in determining the local inertial frame. Not only this result is interesting, but it also has the potential to advance future investigations of the subject.

The basic idea presented in this work (regarding the nature of the observed velocities) can be generalized, in principle, to other observational fields. However, the specific model developed here is applicable only for disk galaxies. Developing other models in order to deal with other observational discrepancies (e.g. velocity dispersions of elliptical galaxies, gravitational lensing, etc) will be the subject of future works.

The discrepancy in galaxy rotation curves was the main reason for developing both modified gravity and dark matter theories. In this work we have shown that the discrepancy can be resolved in a new simple way.

Acknowledgements We would like to thank our dear friends Meir Zeilig-Hess, Oran Ayalon, Dr. Dan Michaels and Dr. Erez Raicher for some valuable advice. In addition, we would like to thank Prof. Stacy McGaugh for sharing with us his data and for some very helpful insights.

\section{Appendix A: OBSERVATIONAL FRAME}

The goal of this appendix is to remind the reader how the measured rotational velocities (the data points in RC's) are derived, and to define one specific system of coordinates relative to which these velocities are given.

When observing a distant object with a spectrograph, using Doppler Effect and spectroscopic techniques, astronomers extract a quantity called 'line-of-sight velocity'. This quantity represents the amount of change in the distance between the observer and the object in one unit of time. When dealing with galaxy rotation curves, line-of-sight velocities for the different areas in a given galaxy are extracted.

The next step is deriving the rotational velocities out of these line-of-sight velocities. This is done by using a formula. This formula, however, contains an implicit assumption. It turns out that the reference frame of the rotational velocities is also (implicitly) determined when using this formula. This is a crucial point and we will get back to it in the next paragraph. Now, actually there are several different methods and several different formulas for this stage. Let us demonstrate the simplest case because it will not change the basic idea. 


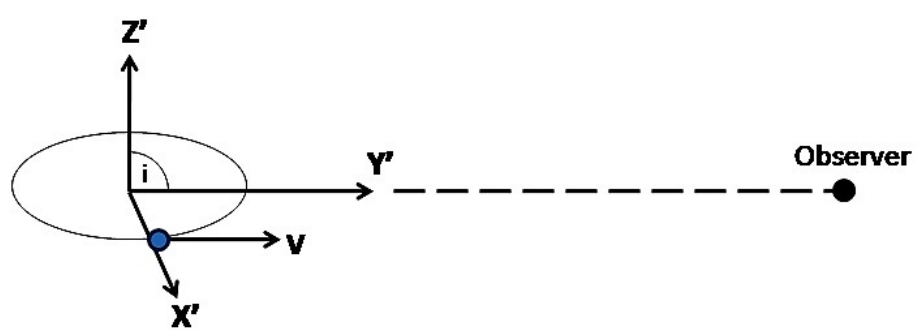

Fig. A.1: A body orbiting a galactic center. In this simple case $v_{s y s}=0$ and $\sin (i)=1$ so the rotational velocity is equal to the line-of-sight measured velocity. This rotational velocity is given relative to a system of coordinates in which the line-of-sight is fixed (as opposed to systems of coordinates where the line-of-sight might rotate around the $z-$ axis)

The simplest formula is given by:

$$
v_{c}(r)=\frac{v-v_{\text {sys }}}{\sin (i)}
$$

where $v_{c}(r)$ is the rotational velocity of the orbiting body, $v$ is the body's line-of-sight velocity, $v_{\text {sys }}$ is the galactic line-of-sight velocity, and $i$ is the inclination angle of the galactic plane ("Edge On" galaxies have $i=90^{\circ}$ ). It is important to emphasize that $v_{c}(r)$ is the velocity which appears in RC's (e.g. the black data points in Fig. 1). Relation A.1 is used only when the observed area is located on the galaxy's main axis.

As noted before, the reference frame (relative to which the rotational velocities $v_{c}(r)$ are given) is also determined when using this relation. Actually, it is not fully determined, it is constrained. Any reference frame in which the line-of-sight to the observer is stationary (i.e. does not rotate) is valid.

To illustrate this, let us focus on the following example in which $v_{s y s}=0$ and $\sin (i)=1$. In this case, relation A.1 states that the rotational velocity is equal to the measured line-of-sight velocity. However, as can be seen in Fig. A.1, this is valid only relative to system $K^{\prime}$ where the observer is stationary. In any reference frame which is rotating relative to $K^{\prime}$ the rotational velocity will take a different value and the observer will not be stationary. The reader may take a minute to be fully convinced at this point.

Now, let us choose one specific reference frame which satisfies the above requirement (i.e. a frame, relative to which the rotational velocities are given). We choose a frame whose fundamental plane coincides with the galaxy plane and its origin coincides with the galactic center. Of course, we also set the frame in such a way that its the line-of-sight to the observer is stationary (in the simple case of "edge on" galaxy, the primary axis is pointing towards the observer). This frame of reference is denoted in the main text as $K^{\prime}$. Our proposition, as was already presented in section 2, is simply the statement that system $K^{\prime}$ is not an inertial system.

\section{Appendix B: ROTATION CURVES PLOTS}

The rest of the fits and a table summarizing the best-fit $M / L$ 's and $\omega$ 's are given below. 
Table 2 The best-fit $M / L$ and $\omega$ for each galaxy

\begin{tabular}{cccccccc}
\hline Galaxy & $\omega^{a}$ & $M / L^{b}$ & $\chi^{2 c}$ & Galaxy & $\omega$ & $M / L$ & $\chi^{2}$ \\
\hline M33 & 1.12 & 0.97 & 118 & NGC 4085 & 4.58 & 0.50 & 3.19 \\
NGC 1003 & 0.75 & 0.54 & 5.56 & NGC 4088 & 1.04 & 1.16 & 1.48 \\
NGC 1560 & 1.82 & 2.57 & 2.38 & NGC 4100 & 1.07 & 2.36 & 2.33 \\
NGC 247 & 1.51 & 1.76 & 3.74 & NGC 4157 & 1.12 & 2.27 & 0.83 \\
NGC 300 & 1.55 & 0.96 & 1.14 & NGC 4183 & 0.81 & 1.45 & 0.1 \\
NGC 3726 & 0.93 & 1.19 & 3.42 & NGC 4217 & 1.73 & 1.72 & 2.99 \\
NGC 3769 & 0.89 & 1.77 & 4.69 & NGC 5033 & 0.74 & 5.06 & 4.44 \\
NGC 3877 & 0.94 & 1.78 & 3.01 & NGC 5585 & 1.50 & 0.87 & 14.6 \\
NGC 3893 & 1.71 & 1.43 & 3.53 & NGC 6946 & 0.72 & 0.79 & 8.76 \\
NGC 3917 & 1.16 & 1.58 & 4.38 & NGC 7793 & 1.49 & 1.40 & 1.22 \\
NGC 3949 & 4.51 & 0.48 & 2.78 & UGC 128 & 0.63 & 3.49 & 1.05 \\
NGC 3953 & 1.40 & 2.25 & 0.69 & UGC 6446 & 0.81 & 1.70 & 1 \\
NGC 3972 & 2.66 & 1.00 & 1.67 & UGC 6667 & 1.47 & 1.74 & 0.83 \\
NGC 3992 & 0.99 & 4.26 & 1.13 & UGC 6818 & 2.86 & 0.16 & 1.03 \\
NGC 4010 & 1.80 & 1.37 & 1.38 & UGC 6917 & 1.28 & 2.01 & 0.94 \\
NGC 4013 & 1.13 & 2.90 & 0.95 & UGC 6930 & 1.17 & 1.30 & 0.52 \\
NGC 4051 & 1.49 & 1.11 & 1.02 & UGC 6983 & 0.80 & 3.11 & 0.87 \\
\hline
\end{tabular}

${ }^{a} \omega$ is given in $\left[10^{-16} \mathrm{rad} / \mathrm{sec}\right]$

${ }^{b}$ The $M / L$ ratios are given in the B-band. The units are $\left[M_{\text {sun }} / L_{\text {sun }}\right]$.

${ }^{c}$ These are the reduced $\chi^{2}$ values of each fit.

\section{References}

Babcock H. W., 1939, Lick Observatory Bulletin 19, 411

Burkert A., 1995, ApJ, 447, L25 12

Carignan C., 1985, ApJ, 299, 59

Carignan C., Chemin L., Huchtmeier W. K., Lockman F. J., 2006, The Astrophysical Journal Letters 641.2, L109 7

Carignan C., Frank B. S., Hess K. M.,Lucero D. M., Randriamampandry T. H., 2013, AJ, 146 7, 8

Corbelli E., Salucci P., 2000, MNRAS, 311.2, 441 7, 8

Einstein A., 1920, Relativity: The special and general theory, Penguin, chapter 42

Freeman K.C., 1970, ApJ, 1606

Geehan J. J.,Fardal M. A. ,Babul A., Guhathakurta P., 2006, MNRAS, 366.3, 9968

Hernquist L., 1989, ApJ, 356, 3596

Jobin M., Carignan C., 1990, AJ, 100, 6487

McGaugh S., 2017, McGaugh's Data Pages. N.p., n.d. Web. 22 Jan. 2017, ¡http://astroweb.case.edu/ssm/data/i, 2, 9

McGaugh S., 2005, ApJ, 632, 8599

Milgrom M., 1983, ApJ,270, 3652

Navarro J., Frenk C., White S., 1997, ApJ, 490, 49312

Puche D., Carignan C., Wainscoat R. J., 1991, AJ, 101, 447 7, 8

Rubin V. C., Ford W. K. Jr, Thonnard N., 1980, ApJ, 238, 4711

Sancisi R., 2004, IAU Symposium, Vol 2209

Sanders R., McGaugh S., 2002,ARA\&A, 40, 2179

Swaters R., Sancisi R., van der Hulst J.M., van Albada T.S., 2012, MNRAS, 425.3, 229912

Toomre, A., 1963, ApJ, 138, 3856 

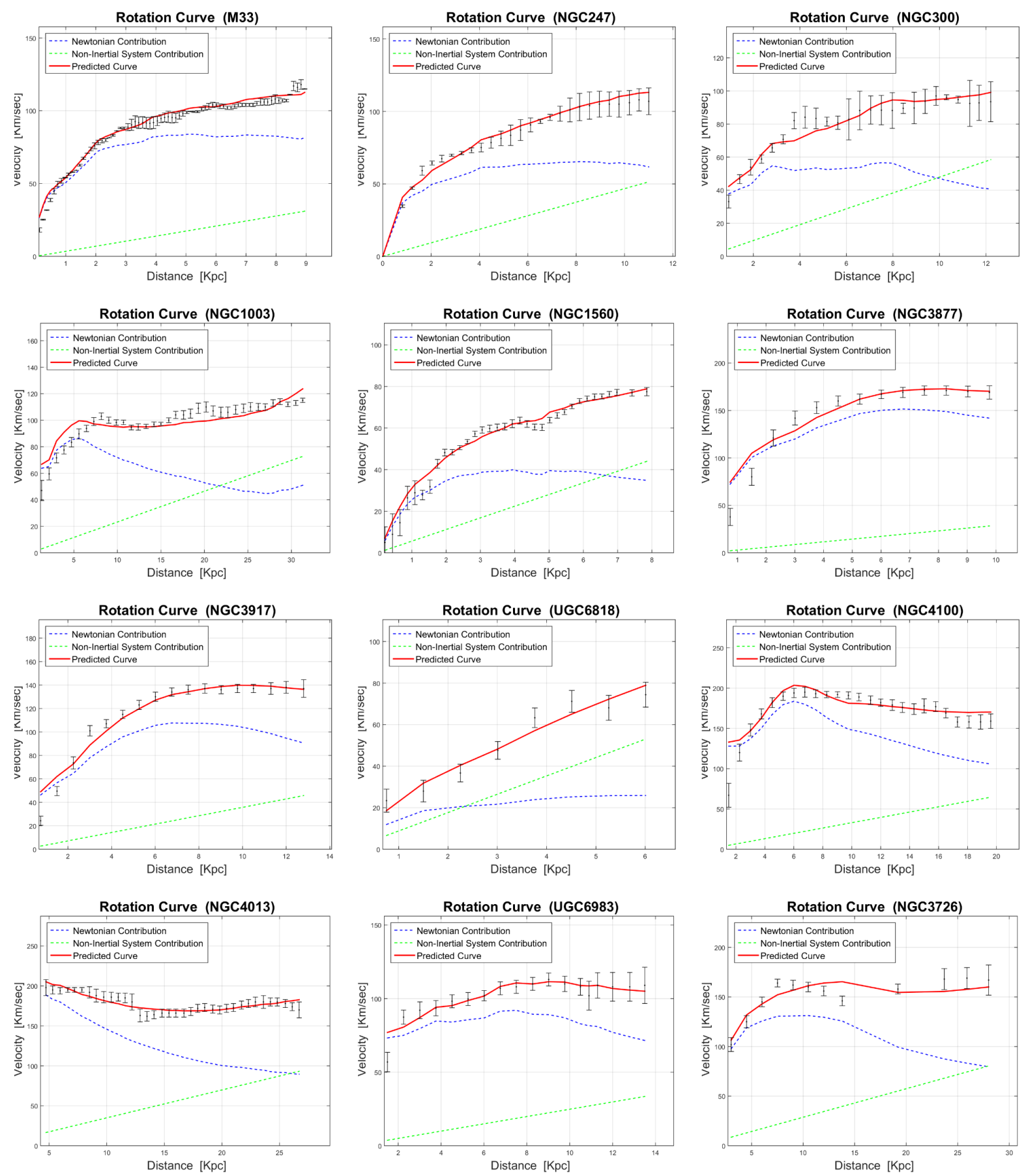

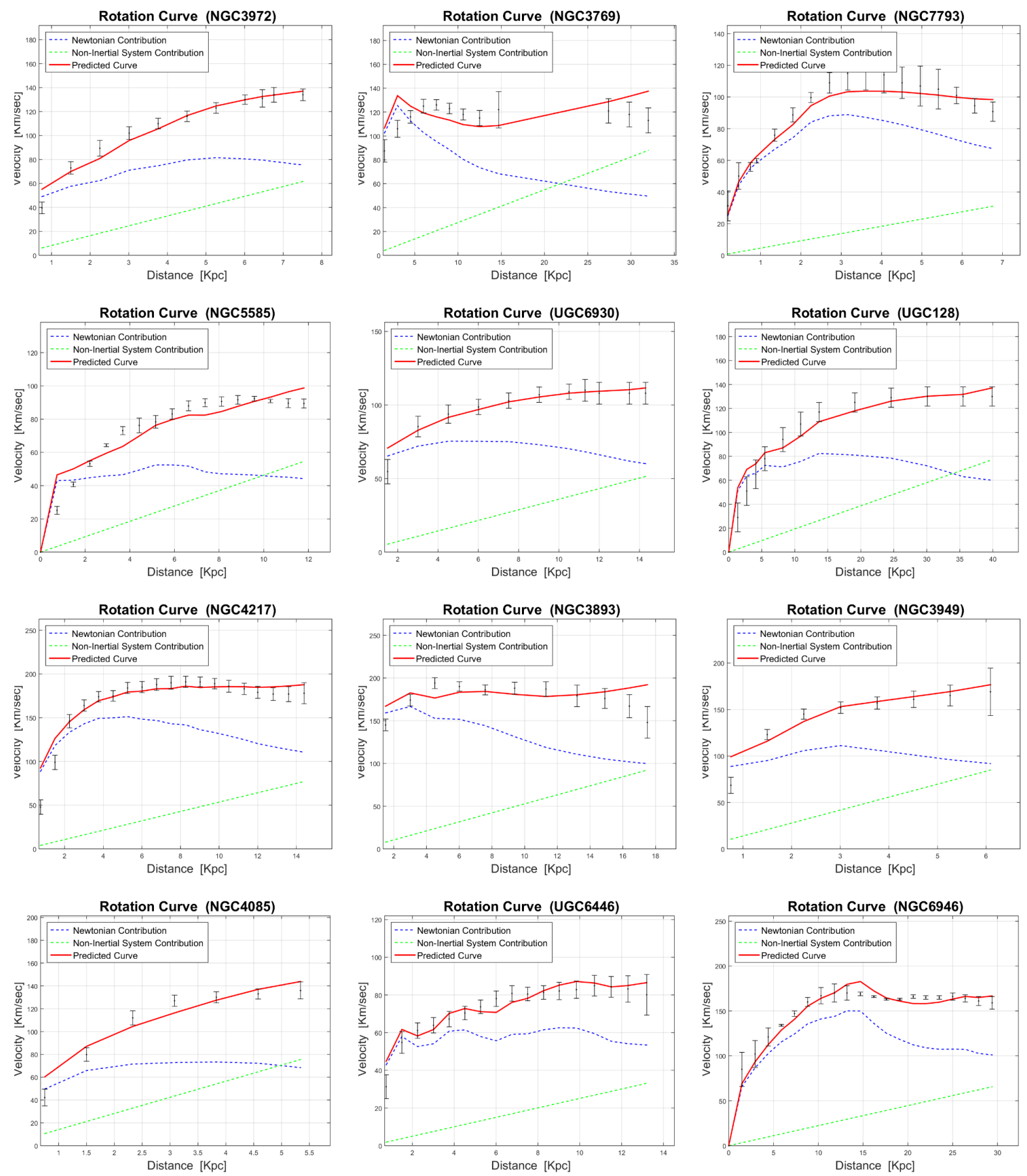

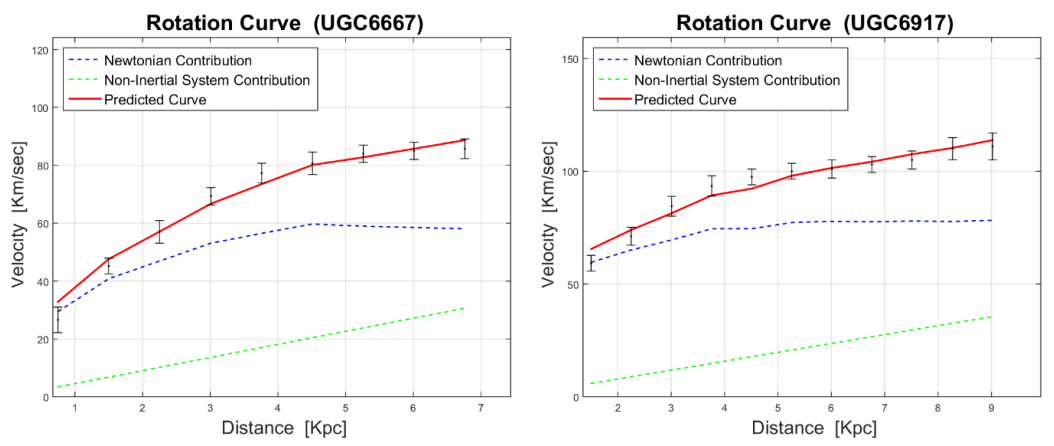

Fig. B.1: RC's for the different galaxies are presented. In each panel, one can see the measured values (black error bars) together with the predicted curve (red line). The blue dashed curve corresponds to the Newtonian term $v_{k}(r)$ while the green dashed line corresponds to the correction term $\omega r$. 